\title{
Evidence of a Synergistic Effect of Acupoint Combination: A Resting-State Functional Magnetic Resonance Imaging Study
}

\author{
Jiping Zhang, MMed, ${ }^{1, *}$ Yu Zheng, MMed, ${ }^{1, *}$ Yanjie Wang, PhD, Shanshan Qu, MMed, \\ Shaoqun Zhang, MMed, ${ }^{1}$ Chunxiao Wu, MMed,' Junqi Chen, PhD, ${ }^{2}$ Huailiang Ouyang, MMed, \\ Chunzhi Tang, $\mathrm{PhD}^{4}$, and Yong Huang, $\mathrm{PhD}^{1}$
}

\begin{abstract}
Objective: This study aimed to find evidence of a synergistic effect of acupoint combinations by analyzing different brain regions activated after acupuncture at different acupoint combinations.

Methods: A total of 57 healthy subjects were randomly distributed into three groups: LR3 plus KI3 acupoints, LR3 plus sham acupoint, or LR3 alone. They underwent a magnetic resonance imaging scan before and after acupuncture. The amplitude of low-frequency fluctuation (ALFF) and regional homogeneity (ReHo) values of different brain regions were analyzed to observe changes in brain function.

Results: ALFF and ReHo produced an activated area in the cerebellum posterior lobe after acupuncture at LR3 plus KI3 acupoints versus LR3 alone. ALFF and ReHo revealed altered activity in Brodmann area 10 (BA10), BA18, and brainstem pons after acupuncture at LR3 plus sham acupoint compared with at LR3 alone. A comparison of acupuncture at LR3 plus KI3 acupoints with LR3 plus sham acupoint demonstrated an increase in BA6 of ALFF and a downregulation of ReHo.

Conclusions: The increased number of brain regions with altered brain activity after acupuncture at acupoint combinations versus a single acupoint are evidence of the synergistic effect of acupoint combinations. BA6 was significantly activated after acupuncture at LR3 plus KI3 acupoints compared with at LR3 plus sham acupoint, suggesting that BA6 is the specific region of synergistic effect of acupoint combinations of LR3 plus KI3 acupoints. Affected brain regions were different between acupuncture at LR3 plus sham acupoint and LR3 alone, which indicates that the sham acupoint may have some psychological effect. However, the specific mechanism of acupoint combinations requires further research.
\end{abstract}

Keywords: acupuncture, acupoints combination, MRI, synergistic effect

\section{Introduction}

A CUPUNCTURE IS AN IMPORTANT part of Traditional Chinese Medicine, and it is widely applied for the treatment of various diseases, such as motor-system and nervous-system diseases. Many randomized controlled trials and evidence-based medicine have fully affirmed the remarkable curative effect of acupuncture on a variety of diseases. ${ }^{1-3}$
Acupoint combinations account for the vast majority of prescribed acupuncture points. Acupoint combination means the use of two or more acupoints at once during acupuncture therapy, and its action can be classified into synergy, antagonism, or other effects (e.g., additive, no interaction, and mixed). Synergy occurs when the effect of an acupoint combination is stronger than the simple sum of the effects of individual acupoints, whereas antagonism means that the

\footnotetext{
${ }^{1}$ School of Traditional Chinese Medicine, Southern Medical University, Guangzhou, China.

${ }^{2}$ Department of Rehabilitation Medicine, Third Affiliated Hospital of Southern Medical University, Guangzhou, China.

${ }^{3}$ Department of Traditional Chinese Medicine, Zhujiang Hospital of Southern Medical University, Guangdong, China.

${ }^{4}$ Clinical Medical College of Acupuncture, Moxibustion and Rehabilitation, Guangzhou University of Chinese Medicine, Guangzhou, China.

*These authors contributed equally to this work.

(C) Jiping Zhang et al., 2016; Published by Mary Ann Liebert, Inc. This Open Access article is distributed under the terms of the Creative Commons License (http://creativecommons.org/licenses/by/4.0), which permits unrestricted use, distribution, and reproduction in any medium, provided the original work is properly credited.
} 
effect of acupoint combinations is weaker than the sum of the effects of individual acupoints using a variety of indicators. ${ }^{4}$ Undoubtedly, synergy of acupoint combinations is a desirable therapeutic effect.

Clinical trials have shown significantly better efficacy of acupoint combinations compared with a single acupoint. ${ }^{5-7}$ Animal experiments demonstrated the efficacy of acupuncture using acupoint combinations at the molecular level. For example, Pan et al. ${ }^{8}$ reported that electroacupuncture (EA) at feishu (BL13) and xinshu (BL15) acupoints protected against pulmonary hypertension by regulating the activity of endothelium-derived endothelin 1 (ET-1) and endothelial nitric oxide synthase (eNOS). Yan et al. ${ }^{9}$ found that EA of the Zhongwan (CV12), Tianshu (ST25), or Shangjuxu (ST37) acupoints alone or in combination relieved acetic acid-induced intestinal mucosal lesions in rats, and the effect of joint application of these three acupoints was significantly better. Nevertheless, the mechanisms of synergy have not been elucidated.

The effect of acupoint combinations can be objectively assessed using measurements of functional brain activity. ${ }^{10}$ Brain regions respond to acupuncture in different acupoint combinations with different activity signals, which are collected, compared, processed, and delivered to target organs. These data demonstrate the compatibility of different acupoint combinations based on various clinical effects.

Currently, functional brain-imaging research focuses on the specificity of meridians and acupoints. Previous studies using functional magnetic resonance imaging (fMRI) compared differences in brain function between acupuncture at a single acupoint and the surrounding (nonacupoint) area ${ }^{11-13}$ or different acupoints. ${ }^{14-17}$ These studies confirmed the specificity of meridians and acupoints in relation to brain function. However, these studies evaluated changes in brain function as a result of a single acupoint, and few previous studies investigated the effects of acupoint combinations. Huang et al. ${ }^{18}$ examined fMRI in healthy people receiving acupuncture at the Waiguan (SJ5) versus Waiguan plus Yanglingquan (GB34) acupoints. They found the acupuncture point combination of SJ5 and GB34 within the hand-foot Shaoyang meridians improved motor and sensory dysfunction and equilibrium disturbances. Nevertheless, these acupuncture studies were based on a block design, that is, stimulation using an "acupuncture-restingacupuncture-resting" scheme, which is not clinically suitable. The present study tested acupuncture in different acupoint combinations using resting-state fMRI, and compared the imaging data to verify changes in brain functional connectivity in different brain regions.

Previous studies demonstrated persistent effects of acupuncture. Vitally, Napadow et al. ${ }^{19}$ observed a linearly decreasing time variation in the activation of sensorimotor brain regions in response to electrostimulation at the acupoint Zusanli (ST36) and a sham point, which suggested classical habituation. Zheng et al. ${ }^{20}$ found that changes in number and intensity were greater at $15 \mathrm{~min}$ after EA stimulation at Yintang and Baihui (GV20) compared to 5 min after needle removal, which demonstrated lasting and strong aftereffects of EA on functional cerebral regions.

The LR3 acupoint has been widely used to study the specificity of meridians and acupoints. Wu et al. ${ }^{21}$ and $\mathrm{Li}$ et al. ${ }^{22}$ certified the specificity of acupoints. This study used
LR3 as a test acupoint to identify possible differences in functional brain changes between acupuncture at LR3 plus KI3 acupoints and LR3 alone on the basis of the previous studies to explain the mechanism of action of acupoint combinations. In addition, since it currently not clear whether all acupoint combinations have synergistic effects, this study also assessed brain activity after acupuncture at LR3 plus a sham acupoint and compared it with the response to acupuncture at LR3 plus KI3 and LR3 alone to identify more evidence of the synergistic effects of acupoint combinations.

The amplitude of low-frequency fluctuation (ALFF) and regional homogeneity (ReHo) were used in this study to measure brain function. ALFF represents the intensity of a blood oxygen level-dependent signal in each voxel, which directly reflects the spontaneous activity of neurons via energy expenditure. ReHo primarily reflects the synchronism of a time series in regional brain areas, not signal intensity, and it indirectly reflects the synchronism of the spontaneous activity of local neurons in a specific brain region. ${ }^{23}$ Therefore, this study evaluated the specificity of LR3 by analyzing alterations in regional brain activities using ReHo and ALFF. The results of two analytical methods were organized and compared with the observed effects of acupuncture on cerebral functional activities to draw more accurate and comprehensive conclusions.

In summary, this study assessed the affected brain regions after acupuncture at different acupoint combinations (LR3 plus KI3 acupoints, LR3 plus sham acupoint, or LR3 alone) using MRI. Changes in brain functional were compared to evaluate possible evidence of synergistic effects of acupoint combinations.

\section{Materials and Methods}

\section{Participants}

Based on the previous report about minimum sample size in neuroimaging studies, ${ }^{24}$ a sample size of 16 per group was needed (total $N=48$ ). Considering a conservative dropout rate of $15 \%$, a total sample size of 57 subjects was determined.

Participants were recruited between September 5, 2012, and January 14, 2013. All of the participants consented to participate in this study and for the case details to be published. Written informed consent was obtained from all participants involved in this study.

Participants were healthy young people from universities and colleges in Guangzhou City, China. The following inclusion criteria were used: aged between 21 and 28 years; never received acupuncture; a regular diet; minimal consumption of liquor, tobacco, tea, and coffee; normal sleep patterns (e.g., going to bed before midnight); moderate weight (body mass index $18.5-23.9 \mathrm{~kg} / \mathrm{m}^{2}$ ); no pain (including dysmenorrhea) or insomnia within a month before the test; and no damaged skin around the test acupoints. All subjects passed a pilot acupuncture test that was performed one month before the study, and all subjects gave full informed consent (Fig. 1)

\section{Random allocation}

This randomized, controlled, single-blind trial was conducted at the First Affiliated Hospital of Guangzhou University of Chinese Medicine. According to complete randomized block design, participants were randomly 
FIG. 1. Consort flowchart.

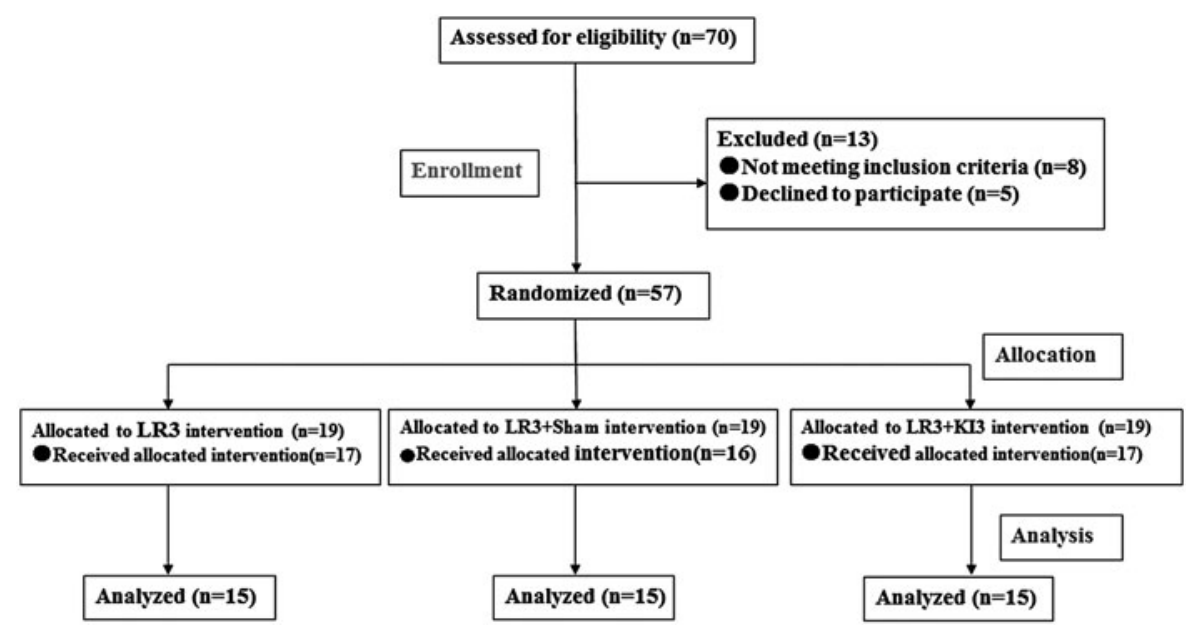

distributed into three groups: LR3 plus KI3 acupoints, LR3 plus a sham acupoint, or LR3 alone. An independent biostatistics professionals generated the random allocation sequence using IBM SPSS Statistics for Windows v19 (IBM Corp., Armonk, NY) and put the random number into opaque sealed envelopes. An investigator who did not participate in the acupuncture interventions controlled the sealed envelopes. Investigators who selected the eligible participants after baseline screening opened the envelopes according to the patients' screening sequence numbers, enrolled participants, and assigned participants to receive interventions.

\section{Blinding}

Due to the procedure of the acupuncture technique, the acupuncture physician in this study was not blinded. Investigators in charge of patient screening and randomized distribution were not involved in intervention and data analyses. The subjects were blinded to the acupoints where they received acupuncture. The acupuncture intervention was performed in a large independent single room with screen dividers for patient blinding and privacy, and subjects' eyes were covered with eyeshades during acupuncture intervention. The statistician, in charge of statistical analysis and independent of acupuncture intervention and data assessor, was blinded to the randomization allocation.

\section{Intervention}

Subjects were asked to pass urine and stool prior to the intervention. Subjects rested for $15 \mathrm{~min}$ at the beginning of the experiment and then underwent the MRI scan and acupuncture. The timeline of acupuncture stimulation and MRI scan is shown in Figure 2.

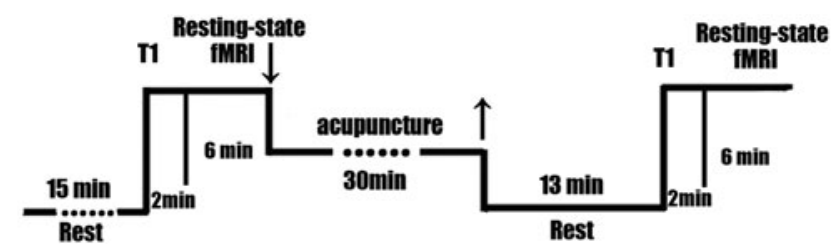

FIG. 2. Trial flowchart.

\section{Acupuncture stimulation}

The same experienced acupuncture physician, who received special training prior to the study to ensure consistent manual acupuncture therapy, performed acupuncture on all subjects. Each subject received acupuncture only once in one of the three combinations: LR3 alone, LR3 plus sham, or LR3 plus KI3. Subjects' eyes were covered with eyeshades so that subjects were blinded to the acupoints where they received acupuncture.

\section{Acupuncture localization}

Acupoints were localized according to the name and location of acupoints (Chinese National Standards GB/T12346; Fig. 3). These were: LR3 - on the dorsum of the foot, in the depression anterior to the junction of the first and second metatarsal bones; KI3 - in the depression between the tip of the media malleolus and tendo calcaneus; and sham pointon the midpoint of the line connecting the anterior superior iliac spine and lateral border of the patella, $2 \mathrm{~cm}$ inside.

\section{Acupuncture operation}

The physician's hands and subjects' skin around the acupoints were sterilized with alcohol before needling. Huatuo needles $(0.30 \times[25-45 \mathrm{~mm}]$; Suzhou Medical Supplies Co., Suzhou, China) were used in this study.

Acupuncture was performed using fingernail press insertion. The order of acupoints was right LR3 $\rightarrow$ left LR3 $\rightarrow$ right $\mathrm{KI} 3 /$ sham point $\rightarrow$ left $\mathrm{KI} 3 /$ sham point. The skin was vertically punctured to a depth of $15 \pm 2 \mathrm{~mm}$. Needles were twirled at an angle of 90-180 and a frequency of 60-90 times/min after a participant sensed the needle. Needles were lifted and thrust in the range of $0.3-0.5 \mathrm{~cm}$ and frequency of 60-90 times/min. Needles were manipulated for $1 \mathrm{~min}$ and held in place for $30 \mathrm{~min}$. The physician manipulated the needle for $1 \mathrm{~min}$ every $10 \mathrm{~min}$ during this $30 \mathrm{~min}$.

\section{fMRI examination}

The fMRI scanning was carried out in a 3.0 Tesla Signa HDxt MRI scanner (GE Company, Fairfield, CT) at First Affiliated Hospital of Guangzhou University of Chinese 

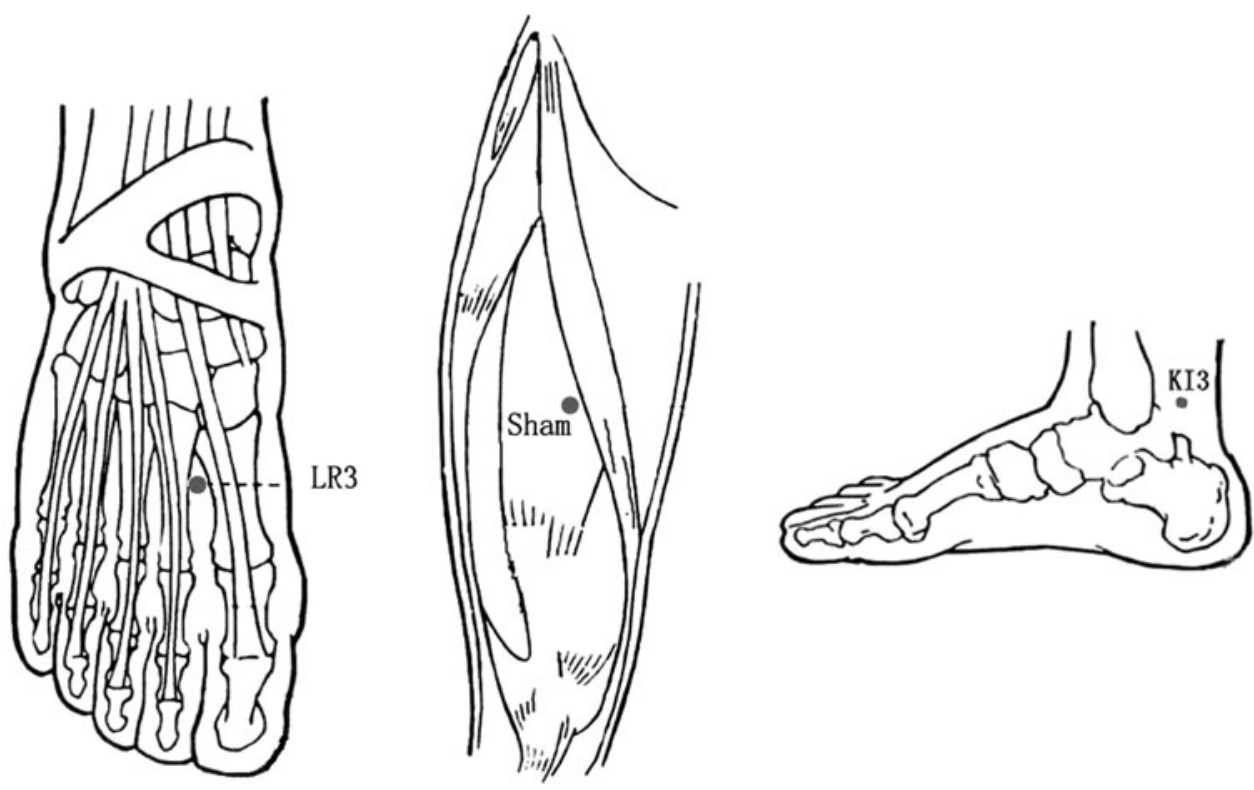

FIG. 3. Location of acupoints.

Medicine. A standard eight-channel phase-array head coil and restraining foam pads were used to minimize head motion.

Subjects were conscious, placed in a supine position, and asked to breathe calmly. Earplugs and a special earshield were used to diminish scanner noise, and eyeshades were used to avoid visual stimulation. During the fMRI scanning, subjects were instructed to move as little as possible and, if they felt uncomfortable, to tell investigators loudly and the scan would be stopped. fMRI scanning began after subjects rested for $15 \mathrm{~min}$.

MRI data (resting-state BOLD sequence) were collected $15 \mathrm{~min}$ before needling and $15 \mathrm{~min}$ after needle withdrawal. Scanning methods were identical between sham and true acupuncture.

(1) Transverse T1-weighted image (T1WI) sequence: $1 \mathrm{~min} 51 \mathrm{sec}$, fast spin echo sequence; OAx T1 FLAIR, repetition time $1750 \mathrm{~ms} /$ echo time $24 \mathrm{~ms}$, inversion time $960 \mathrm{~ms}$, field of view $24 \mathrm{~cm} \times 24 \mathrm{~cm} / Z$, matrix $320 \times 224 /$ number of excitations $=1$, thickness $5.0 \mathrm{~mm} /$ interval $1.0 \mathrm{~mm}, 30$ slices total, echo train length 8 , and bandwidth 31.25 .

(2) Resting-state fMRI BOLD data collection: gradient echo-echo-planar imaging sequence scanning was conducted for $6 \mathrm{~min}$ in accordance with the following parameters: repetition time $3000 \mathrm{~ms} / \mathrm{minimum}$, echo time minimum, flip angle $90^{\circ}$, field of view $240 \mathrm{~mm} \times 240 \mathrm{~mm}$, thickness $5.0 \mathrm{~mm} /$ interval $1.0 \mathrm{~mm}$, 30 slices each time, matrix $96 \times 96 /$ number of excitations $=1$.

\section{Image preprocessing and analytical methods}

Preprocessing was performed using Data Processing Assistant for Resting-State fMRI (DPARSF v2.3; http://rfmri.org/ DPARSF), ${ }^{41}$ which is based on Statistical Parametric Mapping (SPM8; www.fil.ion.ucl.ac.uk/spm) and a Resting-State fMRI Data Analysis Toolkit (REST 1.8; www.restfMRI.net). ${ }^{26}$

The preprocessing procedure includes: (1) convert DICOM to NIFTI; (2) slice timing after removing first 10 time points; (3) realign and exclude subjects with max head motion $>1.5 \mathrm{~mm}$ on any axis and head rotation $>1.5^{\circ}$; (4) co-register T1 to Fun; (5) segment and affixer regularization according to East Asian; (6) normalize by using EPI templates; (7) smooth images with a Gaussian kernel with a isotropic full-width at half-maximum (FWHM) of $4 \mathrm{~mm}$ (data for ReHo analysis without smooth); (8) remove linear detrend; and (9) filter $(0.01-0.08 \mathrm{~Hz})$.

ReHo analysis. ReHo was calculated by REST software. The Kendall's coefficient of concordance (KCC) of each voxel was calculated by the time series of the voxel and its nearest 26 neighboring voxels (cluster size $=27$ ). Then the KCC maps were standardized by dividing their own mean $\mathrm{KCC}$ within the whole brain mask, and the resulting maps were smoothed with a Gaussian kernel with 4 mm FWHM. ${ }^{23,25,26}$

ALFF analysis. ALFF was calculated by REST software. The time series of each voxel was converted to the frequency domain using a fast Fourier transform. Then the square root of the power spectrum was computed and averaged across a predefined frequency interval. The average square root was termed ALFF. ${ }^{27}$ Next, the ALFF was standardized by dividing the mean ALFF within the whole brain mask.

\section{Statistical analysis}

Data were analyzed using REST1.8 software. In the statistical analysis, one-way analysis of variance (ANOVA) was used to explore standardized ALFF/ReHo value differences among the three groups with AlphaSim correction 5 and continuous voxel $>85$. To illustrate clearly the difference between groups, the comparison was further performed on ALFF/ReHo maps using a two-sample $t$-test with Bonfferoni correction $(p<0.0167)$ and continuous voxel $>85$. Finally, ALFF/ReHo value alteration differences among different groups were obtained. Rest1.8 software Viewer was employed to identify the precise anatomical position in the brain with statistical significance on the corresponding MNI coordinate. The results are presented as images. 
Table 1. Participants' Baseline Data

\begin{tabular}{|c|c|c|c|c|c|c|}
\hline \multirow[b]{2}{*}{ Group } & \multirow[b]{2}{*}{$\mathrm{n}$} & \multicolumn{2}{|c|}{ Sex } & \multirow[b]{2}{*}{ Age, years } & \multirow[b]{2}{*}{ Height, $\mathrm{cm}$} & \multirow[b]{2}{*}{ Weight, $\mathrm{kg}$} \\
\hline & & Male & female & & & \\
\hline LR3 & 15 & 9 & 6 & $21.67 \pm 0.488$ & $168.60 \pm 6.811$ & $55.40 \pm 8.348$ \\
\hline LR3 plus sham & 15 & 8 & 7 & $21.47 \pm 0.516$ & $163.80 \pm 7.747$ & $53.87 \pm 6.967$ \\
\hline LR3 plus KI3 & 15 & 7 & 8 & $21.47 \pm 0.516$ & $166.13 \pm 7.482$ & $55.87 \pm 7.586$ \\
\hline
\end{tabular}

\section{Results}

\section{Participants}

Fifty-seven subjects were recruited from the Southern Medicine University and Guangzhou University of Chinese Medicine, China. They were equally allocated into three groups: LR3 alone, LR3 plus sham point, and LR3 plus KI3 acupoints (19 in each group). Two subjects from the LR3 alone group, three subjects from the LR3 plus sham point group and two subjects from the LR3 plus KI3 acupoints group dropped out during the study for the following reasons: could not bear the MRI scanner noise $(n=2)$; noncompliance with study schedule $(n=1)$; and sudden power outages as MRI imaging data acquiring $(n=4)$. Group images from two subjects from the LR3 alone group, three subjects from the LR3 plus sham point group, and two subjects from the LR3 plus KI3 acupoints were excluded during data preprocessing, as max head motion was $>1.5 \mathrm{~mm}$ on any axis and head rotation was $>1.5^{\circ}$. Finally, the fifth subject in each group of images was included for statistical analysis. The baseline and demographics of age, sex, height, and weight with the ITT population are shown in Table 1, which showed that the three groups were comparable at baseline. Furthermore, there was no significant difference between groups in functional imaging before acupuncture according to the REST software.

\section{Brain regions showing changes after acupuncture at the acupoints $L R 3$ plus KI3 versus $L R 3$ alone}

ALFF analysis. Increased ALFF ( $T$ value was positive) was detected in the left superior frontal gyrus (Brodmann area 6 [BA6]), right frontal lobe subgyral corpus callosum (BA32), and subgyral right frontal lobe (BA24). Decreased ALFF ( $\mathrm{T}$ value was negative) was observed in the right middle frontal gyrus (BA10), right cerebellum posterior lobe pyramis, right cerebellum posterior lobe, and right inferior semilunar lobule (Table 2 and Fig. 4).

ReHo analysis. Increased ReHo was detected in the right superior parietal lobule (BA7), right frontal lobe precentral gyrus (BA4), right temporal subgyral lobe (BA22), right cerebellum posterior lobe, left occipital lobe, and cuneus (BA19, 18). Decreased ReHo was observed in the left middle temporal gyrus (BA21; Table 3 and Fig. 5).

Table 2. Brain Areas with ALFF Alteration

\begin{tabular}{|c|c|c|c|c|c|c|c|c|}
\hline \multirow[b]{2}{*}{ Comparisons } & \multirow{2}{*}{$\begin{array}{l}\text { Number } \\
\text { of voxels }\end{array}$} & \multirow[b]{2}{*}{ Brain areas } & \multirow{2}{*}{$\begin{array}{c}\text { Right/ } \\
\text { left }\end{array}$} & \multirow{2}{*}{$\begin{array}{l}\text { Brodmann } \\
\quad \text { area }\end{array}$} & \multicolumn{3}{|c|}{ Talairach $(\mathrm{mm})$} & \multirow[b]{2}{*}{$T$} \\
\hline & & & & & $X$ & $Y$ & $Z$ & \\
\hline \multirow{6}{*}{$\begin{array}{l}\text { LR3 plus KI3 } \\
\text { vs. LR3 }\end{array}$} & 122 & Superior frontal gyrus & $\mathrm{L}$ & 6 & -3 & 3 & 63 & 4.7498 \\
\hline & 849 & Frontal lobe, sub-gyral, corpus callosum & $\mathrm{R}$ & 32 & 15 & 24 & 21 & 4.6052 \\
\hline & 85 & Frontal lobe, sub-gyral & $\mathrm{R}$ & 24 & 21 & 3 & 36 & 3.7494 \\
\hline & 148 & Middle frontal gyrus & $\mathrm{R}$ & 10 & 42 & 54 & 12 & -3.6715 \\
\hline & 138 & Cerebellum, posterior lobe, pyramis & $\mathrm{R}$ & & 6 & -90 & -33 & 3.8422 \\
\hline & 248 & $\begin{array}{l}\text { Cerebellum posterior lobe, } \\
\text { inferior semi-lunar lobule }\end{array}$ & $\mathrm{R}$ & & 36 & -63 & -54 & -3.5093 \\
\hline \multirow{8}{*}{$\begin{array}{l}\text { LR3 plus sham } \\
\text { vs. LR3 }\end{array}$} & 135 & Superior frontal gyrus & $\mathrm{L}$ & 8 & 0 & 30 & 48 & 3.974 \\
\hline & 93 & Middle frontal gyrus & $\mathrm{L}$ & 9 & -51 & 21 & 33 & 3.9274 \\
\hline & 121 & Occipital lobe, cuneus & $\mathrm{L}$ & 18 & -15 & -99 & 0 & -3.4428 \\
\hline & 282 & Left brainstem, pons & $\mathrm{L}$ & & -15 & -21 & -27 & 4.2434 \\
\hline & 361 & Frontal lobe, frontal forceps & $\mathrm{R}$ & 9 & 18 & 24 & 21 & 4.9586 \\
\hline & 118 & Superior frontal gyrus & $\mathrm{R}$ & 10 & 9 & 63 & 12 & 3.9919 \\
\hline & 103 & Sub-lobar, extra-nuclear & $\mathrm{R}$ & 37 & 27 & -36 & 12 & 3.7882 \\
\hline & 153 & Occipital lobe, cuneus & $\mathrm{R}$ & 18 & 12 & -78 & 12 & -4.8673 \\
\hline \multirow{7}{*}{$\begin{array}{l}\text { LR3 plus KI3 } \\
\text { vs. LR3 plus } \\
\text { sham }\end{array}$} & 575 & Frontal lobe, paracentral lobule & $\mathrm{L}$ & 6 & -3 & -33 & 69 & 5.0682 \\
\hline & 300 & Occipital lobe, cuneus & $\mathrm{L}$ & 18 & -3 & -102 & -3 & 4.07 \\
\hline & 113 & Limbic lobe, posterior cingulate & $\mathrm{L}$ & 18 & 18 & -66 & 3 & -4.8673 \\
\hline & 1196 & Inferior frontal gyrus & $\mathrm{R}$ & 10,9 & 48 & 9 & 22 & -6.9094 \\
\hline & 261 & $\begin{array}{l}\text { Cerebellum posterior lobe, } \\
\text { cerebelum_Crus2_R }\end{array}$ & $\mathrm{R}$ & & 9 & -93 & -30 & 4.618 \\
\hline & 201 & Cerebellum posterior lobe, uvula & $\mathrm{R}$ & & 15 & -69 & -42 & -3.8729 \\
\hline & 770 & Right brainstem, midbrain & $\mathrm{R}$ & & 3 & -33 & -18 & -5.2822 \\
\hline
\end{tabular}

ALFF, amplitude of low-frequency fluctuation. 

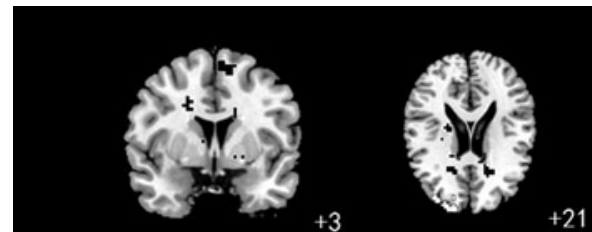

$+21$

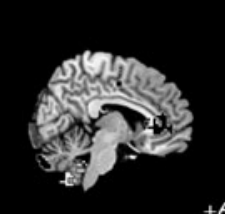

$+6$

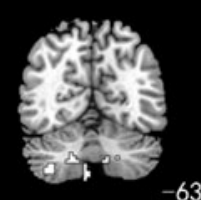

LR3 Plus KI3 VS LR3

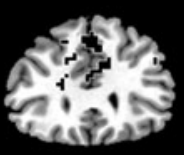

$+30$

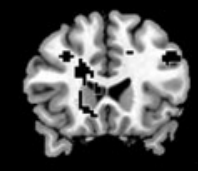

$+22$

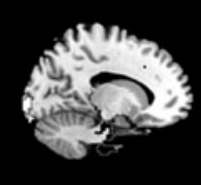

$-15$

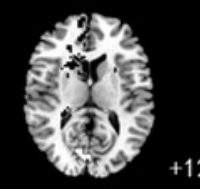

LR3 Plus Sham VS LR3

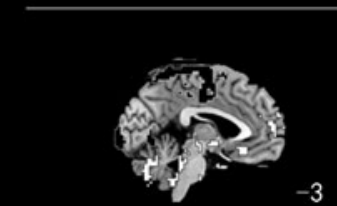

$-3$

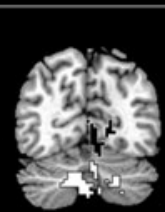

$-68$

LR3 Plus KI3 VS LR3 Plus Sham
FIG. 4. Brain areas with amplitude of low-frequency fluctuation alteration. White areas with black line represent deactivation; black areas with white line represent activation.
Brain regions with changes after acupuncture at the LR3 plus sham acupoints versus LR3 alone

ALFF analysis. ALFF apparently increased in the left superior frontal gyrus (BA8), left middle frontal gyrus (BA9), left brainstem pons, right frontal forceps (BA9), right superior frontal gyrus (BA10), and right sublobar extranuclear (BA37) and decreased in the left and right occipital lobe cuneus (BA18; Table 2 and Fig. 4).

ReHo analysis. Increased ReHo was detected in the left superior occipital gyrus (BA39 and BA19) and left inferior frontal gyrus (BA46). Decreased ReHo was observed in the left superior temporal gyrus (BA22), left medial frontal gyrus (BA24 and BA6), left occipital lobe cuneus (BA18), right middle frontal gyrus (BA10), and right brainstem pons (Table 3 and Fig. 5).

\section{Brain regions with changes after acupuncture} at the LR3 plus KI3 acupoints versus LR3 plus sham

ALFF analysis. Increased ALFF was detected in the left frontal lobe paracentral lobule (BA6), left occipital lobe cuneus (BA18), and right cerebellum posterior lobe cerebelum_crus2_R. Decreased ALFF was observed in the left limbic posterior cingulate lobe (BA18), right inferior frontal gyrus (BA10 and BA9), right cerebellum posterior lobe uvula, and right brainstem midbrain (Table 2 and Fig. 4).

Table 3. Brain Areas with ReHo Alterations

\begin{tabular}{|c|c|c|c|c|c|c|c|c|}
\hline \multirow[b]{2}{*}{ Comparisons } & \multirow{2}{*}{$\begin{array}{l}\text { Number } \\
\text { of voxels }\end{array}$} & \multirow[b]{2}{*}{ Brain areas } & \multirow{2}{*}{$\begin{array}{c}\text { Right/ } \\
\text { left }\end{array}$} & \multirow{2}{*}{$\begin{array}{l}\text { Brodmann } \\
\text { area }\end{array}$} & \multicolumn{3}{|c|}{ Talairach (mm) } & \multirow[b]{2}{*}{$T$} \\
\hline & & & & & $X$ & $Y$ & $Z$ & \\
\hline \multirow{6}{*}{$\begin{array}{l}\text { LR3 plus KI3 } \\
\text { vs. LR3 }\end{array}$} & 106 & Superior parietal lobule & $\mathrm{R}$ & 7 & 15 & -72 & 51 & 4.6556 \\
\hline & 146 & Frontal lobe, precentral gyrus & $\mathrm{R}$ & 4 & 27 & -27 & 66 & 4.5514 \\
\hline & 89 & Temporal lobe, sub-gyral & $\mathrm{R}$ & 22 & 36 & -63 & 6 & 4.1554 \\
\hline & 86 & Cerebellum posterior lobe & $\mathrm{R}$ & & 9 & -87 & -39 & 3.9505 \\
\hline & 138 & Occipital lobe, cuneus & $\mathrm{L}$ & 19,18 & -3 & -84 & 24 & 4.4965 \\
\hline & 93 & Middle temporal gyrus & $\mathrm{L}$ & 21 & -66 & -36 & -18 & -4.0062 \\
\hline \multirow{7}{*}{$\begin{array}{l}\text { LR3 plus sham } \\
\text { vs. LR3 }\end{array}$} & 91 & Superior occipital gyrus & $\mathrm{L}$ & 39,19 & -33 & -75 & 24 & 4.7224 \\
\hline & 87 & Inferior frontal gyrus & $\mathrm{L}$ & 46 & -48 & 42 & 9 & 3.7627 \\
\hline & 161 & Superior temporal gyrus & $\mathrm{L}$ & 22 & -36 & -12 & -15 & -4.7235 \\
\hline & 103 & Medial frontal gyrus & $\overline{\mathrm{L}}$ & 24,6 & -12 & -21 & 57 & -4.4383 \\
\hline & 96 & Occipital lobe, cuneus & $\mathrm{L}$ & 18 & -18 & -99 & -3 & -4.0951 \\
\hline & 86 & Middle frontal gyrus & $\mathrm{R}$ & 10 & 36 & 48 & -9 & -5.0098 \\
\hline & 88 & Right brainstem, pons & $\mathrm{R}$ & & 6 & -33 & -33 & -3.5016 \\
\hline \multirow{4}{*}{$\begin{array}{l}\text { LR3 plus KI3 } \\
\text { vs. LR3 plus } \\
\text { sham }\end{array}$} & 85 & Sub-lobar, extra-nuclear & $\mathrm{R}$ & & 3 & -12 & -6 & 4.1944 \\
\hline & 94 & Frontal lobe, precentral gyrus & $\mathrm{R}$ & 6 & 66 & -3 & 30 & -4.3068 \\
\hline & 130 & Occipital lobe, lingual gyrus & $\mathrm{R}$ & 19 & 18 & -84 & -15 & -4.0172 \\
\hline & 110 & Middle temporal gyrus & $\mathrm{R}$ & 21 & 72 & -21 & 3 & -3.8863 \\
\hline
\end{tabular}

ReHo, regional homogeneity. 


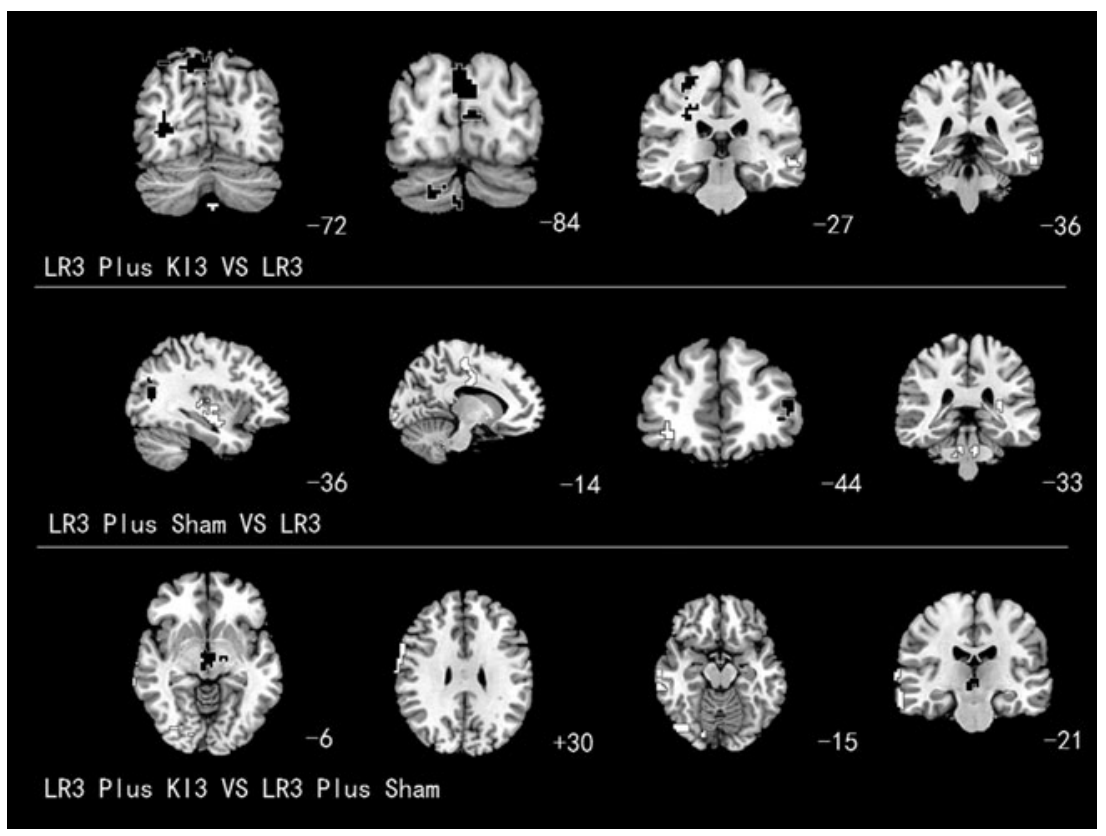

FIG. 5. Brain areas with regional homogeneity alteration. White areas with black line represent deactivation; black areas with white line represent activation.

ReHo analysis. Increased ReHo was detected in the right sublobar extranuclear region. Decreased ReHo was observed in the right frontal lobe precentral gyrus (BA6), right occipital lobe lingual gyrus (BA19), and right middle temporal gyrus(BA21; Table 3 and Fig. 5).

\section{Discussion}

Comparison of LR3 plus KI3 acupuncture versus LR3 alone revealed that ALFF alterations were concentrated in BA6, BA10, BA24, BA32, the cerebellum posterior lobe, and inferior semilunar lobule regions of the brain. ReHo alterations were observed in BA4, BA7, BA18, BA19, $\mathrm{BA} 21, \mathrm{BA} 22$, and cerebellum posterior lobe regions of the brain. The ALFF and ReHo results demonstrated that cerebellum posterior lobe regions were activated brains areas. The simultaneously increased values of ALFF and ReHo demonstrated that the local neuronal activity of cerebellum posterior lobe regions was enhanced, and the surrounding neuronal activity was coordinated more closely. The cerebellum posterior lobe maintains body balance, participates in the initial processing of sensory and emotional information, and regulates neurological function. ${ }^{28}$ The activity of the posterior lobe of the cerebellum has a modulatory effect in the treatment of ventricular hypertrophy, ${ }^{29}$ which suggests that acupuncture at the acupoints LR3 plus KI3 strengthens the function of the cerebellar posterior lobe. Movement disorders often involve impaired functioning of the cerebellum, which can be hypo-or hyperactive compared with healthy functioning. ${ }^{30}$ So the increased cerebellum common activity indicates acupuncture at LR3 plus $\mathrm{KI} 3$ acupoint combination can be used to treat movement diseases such as tremor, dystonia, and so on.

Previous studies found that acupuncture at LR3 specifically activates BA7, BA18, BA19, and other brain regions. $^{22,31}$ BA7, BA18, and BA19 are involved in the formation of visual transduction, and one study showed that BA7 and the posterior cingulate were the information- processing hubs that connect the dorsal cochlear nucleus and ventral palladium. ${ }^{32}$ In addition, stimulation of LR3 also activated the thalamus and the limbic system. These areas were involved in visceral modulation. ${ }^{15}$ Therefore, acupuncture at LR3 could be applied to the treatment of visionrelated diseases as well as visceral pain and body paralysis. Chen et al. found that acupuncture at KI3 enhanced active connections of the post-temporal cortex, dorsolateral prefrontal cortex, and the ventromedial prefrontal cortex. ${ }^{33}$ Zhong et al. ${ }^{34}$ found that acupuncture at KI3 increased incoming and outgoing activity of local superior temporal gyrus neurons and strengthened the connection of the superior temporal gyrus with the posterior central gyrus.

The present study found brain regions associated with visual and auditory perception, body movement, and association are selectively activated after acupuncture at KI3. Acupuncture at $\mathrm{KI} 3$ acts on certain brain regions related to its clinical application, as acupuncture at KI3 is usually used in the treatment of developmental retardation, presenility, diseases of the urogenital system, headache, auditory disorders, cognitive dysfunctions, insomnia, chronic cough, and chronic diarrhea, according to Traditional Chinese Medicine. ${ }^{35}$ The post-temporal cortex and superior temporal gyrus are closely related to the formation of auditory sense. Therefore, KI3 is widely used in the treatment of hearingrelated diseases. The functional activities of visual centerrelated brain areas (BA7, BA18, BA19, and BA41) and auditory event-related brain regions (BA21 and BA22) are activated with the combination of LR3 and KI3, which increases the related neuronal spontaneous activity levels of vision and hearing. Therefore, the LR3 and KI3 combination could be more beneficial than the single acupoint in the treatment of audiovisual diseases. The increased number of activated brain regions confirms that the combining of acupoints has a synergistic effect on the central nervous system. Huang et al. ${ }^{18}$ and Leung et al. ${ }^{36}$ also confirmed that combining acupoints produces a synergistic effect using functional brain imaging techniques. 
Acupuncture at LR3 plus sham produced ALFF alterations in BA8, BA9, BA10, BA18, BA37, and brainstem pons regions. ReHo produced affects in the BA6, BA10, BA18, BA19, BA22, BA24, BA46, and brainstem pons regions. ALFF and ReHo both showed that the common affected areas were BA10, BA18, and brainstem pons regions. BA10 is located in the prefrontal lobe, and it participates in association and management function. A previous study showed that BA10 strengthened the functional connectivity of amygdaloidal nucleus, anterior cingulate, and superior frontal gyrus cortex when subjected to continuous pressure. ${ }^{37}$ Another study found that the ALFF value of BA10 was significantly higher in patients with post-traumatic stress disorder. ${ }^{38}$ BA10 ALFF value increased and ReHo values decreased as a result of the combination of the LR3 plus sham acupoints, which suggests that the local neuronal activity of BA10 increased, but the surrounding neuronal activity is not consistent with the changes in BA10. These data indicate that this effect may be the result of differences in the positioning and de qi sensation of the nonacupuncture point and the true acupoint, which caused subjects to generate relevant associations. Therefore, the functional activity changes in BA10 may be the result of the sham acupoint's influence on the acupoint. In contrast, the ALFF value of BA10 decreased in the combination KI3 plus LR3. Both acupoint combinations affected the functional activities of BA10, but the roles of the results were different, which may also be the crucial evidence of synergy of this acupoint combination. A lower value of ALFF and ReHo means that the relative signal of local oxygen was reduced, which breaks the connection with surrounding neurons. These results may be a sign of neuronal inhibition. ${ }^{39}$ Inhibition of BA18 means that vision-related neuronal activity levels decrease as a result of the LR3 plus sham combination. These data are consistent with a previous study. ${ }^{40}$ ReHo values of BA18 decreased as a result of the LR3 plus sham combination, whereas the ReHo value increased under the influence of the LR3 plus KI3 combination. This phenomenon may be the central difference between the combinations LR3 plus KI3 and LR3 plus sham. However, it may be a normal effect of adjustment (excitatory and inhibitory). It is possible that acupoint combinations have novel central effects, and nonacupoints (sham) impact LR3, which does not correspond to a $1+0=1$ relationship. The sham acupoint impacts the central mechanisms of LR3, but their specific interactions require further in-depth research.

Comparisons of acupuncture at the LR3 plus KI3 acupoints to the LR3 plus sham revealed that ALFF alterations were concentrated in BA6, BA9, BA10, BA18, and the brainstem midbrain, cerebellum crus, and cerebellar posterior lobe regions of the brain. ReHo alterations were observed in BA6, BA19, BA21, and the sublobar and extranuclear regions of the brain. The ALFF and ReHo results demonstrated that the common activated brain region was BA6, which exhibited increases in ALFF and decreases in ReHo. Enhanced ALFF means an increase in regional blood oxygen signal intensity, which is associated with more local field potentials and multiunit activities. A smaller value of ReHo indicates lower regional synchronization. Synchronization reflects intrinsic coherent neuronal activity within spatially organized brain regions, which may be relevant to neural inactivity in a regional brain area. ${ }^{23,26,41}$ BA6 is a motor cortical area in the posterior frontal lobe that is directly anterior to the primary motor cortex. This area participates in the planning and execution of volitional movement. BA6 receives significantly higher regional cerebral blood flow across pain modalities. ${ }^{42}$ Therefore, it was hypothesized that acupuncture at the LR3 plus KI3 acupoints have a synergistic effect on pain. Acupuncture at the LR3 plus KI3 acupoints is widely used to regulate blood pressure. Cell et al. ${ }^{43}$ showed that high blood-pressure levels were associated with a smaller gray-matter volume in the supplementary motor area (BA6). Another study suggested that changes in the infrared radiation temperature of the lower extremity acupoints KI3 and LR3 are closely related to the pathological characteristics of the dysfunction of conception and thoroughfare vessels syndrome of hyperplasia of the mammary gland. ${ }^{44}$ Therefore, changes in BA6 function after acupuncture at the LR3 plus KI3 acupoints are evidence of a synergistic effect of this acupoint combination.

Furthermore, BA18, BA19, BA21, and visual association areas were inactivated, which are signs of visual neuronal inhibition. Previous studies confirmed that acupuncture at LR3 induced specific patterns of activities in visual association brain areas. ${ }^{18,25}$ Common activated areas were found in response to LR3 plus KI3 or LR3 plus sham in visual association brain areas. Therefore, this phenomenon represents a synergistic effect of this acupoint combination, which is an extension of the individual acupoint effects.

This study has some limitations. All subjects in this study were healthy. Previous research demonstrated that signal activation patterns during the same acupuncture procedure are different between healthy subjects and ill patients. ${ }^{21,45}$ Accordingly, the results could only be tentatively presumed useful for the treatment of relevant diseases. A further study will assess the mechanisms of acupoints combinations in pathological conditions.

\section{Conclusion}

The increased number of altered brain regions as a result of acupuncture at the LR3 plus KI3 acupoints versus LR3 alone supports a synergistic effect of acupoint combinations. BA6 was activated more strongly after acupuncture at the LR3 plus KI3 acupoints compared with LR3 plus sham, which suggests that BA6 is one of the specific brain regions for the LR3 plus KI3 combination. Some additional alterations were observed in brain regions after acupuncture at LR3 plus sham compared with acupuncture at the LR3 acupoint alone. These data suggest that changes in brain activity after acupuncture at LR3 are affected by the sham acupoint, but the mechanisms of their interaction need further research.

\section{Acknowledgments}

This study was approved by the Institutional Review Boards of the Chinese Ethics Committee of Registering Clinical Trials (www.chictrdb.org/; identifier: ChiECRCT2012011) and registered at www.chictr.org/en/ (identifier: ChiCTR-TRC-12002427). The study was performed according to the principles of the Declaration of Helsinki (Edinburgh version 2000).

This study was supported by the National Key Basic Research and Development Project (973 Program), grant No. 2012CB518504; URL: http://www.nsfc.gov.cn/ Manager: 
Chunzhi Tang; the National-level Undergraduate Student Innovation Venture Training Project of Local Colleges, No. 201212121048; Manager: Chunxiao Wu; the Three-stage Key Subject Construction Project of Guangdong Province of China (211 Project), No. Yuefagaishe (2009)431.

We are very grateful to the healthy volunteers and staff from the MRI Center of the First Affiliated Hospital of Guangzhou University of Chinese Medicine in China.

\section{Author Disclosure Statement}

No competing financial interests exist.

\section{References}

1. Lee HS, Park HL, Lee SJ, et al. Scalp acupuncture for Parkinson's disease: a systematic review of randomized controlled trials. Chin J Integr Med 2013;19:297-306.

2. Kim YD, Heo I, Shin BC, et al. Acupuncture for posttraumatic stress disorder: a systematic review of randomized controlled trials and prospective clinical trials. Evid Based Complement Alternat Med 2013;2013:615857.

3. Yan X, Zhu T, Ma C, et al. A meta-analysis of randomized controlled trials on acupuncture for amblyopia. Evid Based Complement Alternat Med 2013;2013:648054.

4. Zhong F, Zeng F, Zheng H, et al. Present study on antagonistic effect of acupoints compatibility. Zhongguo Zhen Jiu 2011;31:1093-1096.

5. Alizadeh R, Esmaeili S, Shoar S, et al. Acupuncture in preventing postoperative nausea and vomiting: efficacy of two acupuncture points versus a single one. J Acupunct Meridian Stud 2014;7:71-75.

6. Ma YX, Ye XN, Liu CZ, et al. A clinical trial of acupuncture about time-varying treatment and points selection in primary dysmenorrhea. J Ethnopharmacol 2013;148: 498-504.

7. Xu HY, Yang F, Zhu J. Effect of electroacupuncture at Hegu (LI 4) and Sanyinjiao (SP 6) on short-term adverse effects of drug-induced abortion. Zhongguo Zhen Jiu 2007; 27:103-105.

8. Pan $\mathrm{P}$, Zhang $\mathrm{X}$, Qian $\mathrm{H}$, et al. Effects of electroacupuncture on endothelium-derived endothelin-1 and endothelial nitric oxide synthase of rats with hypoxia-induced pulmonary hypertension. Exp Biol Med 2010;235:642-648.

9. Yan LP, Ji LX, Wang HJ, et al. Selection of basic acupoints combined with "enteral-disorder-formuia" for electroacupuncture treatment of intestinal mucosal lesion in rats Zhen Ci Yan Jiu 2012;37:458-463.

10. Lai XS, Huang Y. A cerebral functional definition on the specificity of acupoints, needling sensation and association of acupoints based on the "acupoints-brain relation hypothesis." Zhongguo Zhen Jiu 2007;27:777-780.

11. Chen J, Wang J, Huang Y, et al. Modulatory effect of acupuncture at Waiguan (TE5) on the functional connectivity of the central nervous system of patients with ischemic stroke in the left basal ganglia. PLOS ONE 2014; 9:e96777.

12. Li L, Qin W, Bai L, et al. Exploring vision-related acupuncture point specificity with multivoxel pattern analysis. Magn Reson Imaging 2010;28:380-387.

13. Wu Y, Jin Z, Li K, et al. Effect of acupuncture on the brain in children with spastic cerebral palsy using functional neuroimaging (fMRI). J Child Neurol 2008;23:1267-1274.
14. Claunch JD, Chan ST, Nixon EE, et al. Commonality and specificity of acupuncture action at three acupoints as evidenced by fMRI. Am J Chin Med 2012;40:695-712.

15. Liu $\mathrm{H}, \mathrm{Xu} \mathrm{JY}, \mathrm{Li} \mathrm{L}$, et al. fMRI evidence of acupoints specificity in two adjacent acupoints. Evid Based Complement Alternat Med 2013;2013:932581.

16. Tang HT, Wang H, Zhu B, et al. Comparative study on fMRI of the brain based on Dicang (ST 4), Hegu (LI 4) and Houxi (SI 3) on human body. Zhongguo Zhen Jiu 2011;31:521-525.

17. Xue T, Bai L, Chen S, et al. Neural specificity of acupuncture stimulation from support vector machine classification analysis. Magn Reson Imaging 2011;29:943-950.

18. Huang Y, Li TL, Lai XS, et al. Functional brain magnetic resonance imaging in healthy people receiving acupuncture at Waiguan versus Waiguan plus Yanglingquan points: a randomized controlled trial. Zhong Xi Yi Jie He Xue Bao 2009; 7:527-531.

19. Napadow V, Dhond R, Park K, et al. Time-variant fMRI activity in the brainstem and higher structures in response to acupuncture. Neuroimage 2009;47:289-301.

20. Zheng Y, Qu S, Wang N, et al. Post-stimulation effect of electroacupuncture at Yintang (EX-HN3) and GV20 on cerebral functional regions in healthy volunteers: a resting functional MRI study. Acupunct Med 2012;30:307-315.

21. Wu Y, Jin Z, Li K, et al. Functional magnetic resonance imaging activation of the brain in children: real acupoint versus sham acupoint. J Child Neurol 2010;25:849-855.

22. Li L, Liu H, Li YZ, et al. The human brain response to acupuncture on same-meridian acupoints: evidence from an fMRI study. J Altern Complement Med 2008;14:673-678.

23. Zang Y, Jiang T, Lu Y, et al. Regional homogeneity approach to fMRI data analysis. Neuroimage 2004;22:394-400.

24. Friston K. Ten ironic rules for non-statistical reviewers. Neuroimage 2012;61:1300-1310.

25. Liu CH, Ma X, Li F, et al. Regional homogeneity within the default mode network in bipolar depression: a resting-state functional magnetic resonance imaging study. PLoS One. 2012;7(11):e48181.

26. Song XW, Dong ZY, Long XY, et al. REST: a toolkit for resting-state functional magnetic resonance imaging data processing. PLOS ONE 2011;6:e25031.

27. Chen JD, Liu F, Xun GL, et al. Early and late onset, firstepisode, treatment-naive depression: same clinical symptoms, different regional neural activities. J Affect Disord 2012;143:56-63.

28. Smith KA, Ploghaus A, Cowen PJ, et al. Cerebellar responses during anticipation of noxious stimuli in subjects recovered from depression. Functional magnetic resonance imaging study. Br J Psychiatry 2002;181:411-415.

29. Allen G, Buxton RB, Wong EC, et al. Attentional activation of the cerebellum independent of motor involvement. Science 1997;275:1940-1943.

30. Vlaar MP, Mugge W, Groot PF, et al. Targeted brain activation using an MR-compatible wrist torque measurement device and isometric motor tasks during functional magnetic resonance imaging. Magn Reson Imaging 2016;34: 795-802.

31. Yan B, Li K, Xu J, et al. Acupoint-specific fMRI patterns in human brain. Neurosci Lett 2005;383:236-240.

32. Whittingstall K, Bernier M, Houde JC, et al. Structural network underlying visuospatial imagery in humans. Cortex 2014;56:85-98.

33. Chen SJ, Meng L, Yan H, et al. Functional organization of complex brain networks modulated by acupuncture at dif- 
ferent acupoints belonging to the same anatomic segment. Chin Med J (Engl) 2012;125:2694-2700.

34. Zhong C, Bai L, Dai R, et al. Modulatory effects of acupuncture on resting-state networks: a functional MRI study combining independent component analysis and multivariate Granger causality analysis. J Magn Reson Imaging 2012; 35:572-581.

35. Zhu B, Wang Y, Zhang G, et al. Acupuncture at KI3 in healthy volunteers induces specific cortical functional activity: an fMRI study. BMC Complement Altern Med 2015;15:361.

36. Leung A, Zhao Y, Shukla S. The effect of acupuncture needle combination on central pain processing - an fMRI study. Mol Pain 2014;10:23.

37. Tryon MS, Carter CS, Decant R, et al. Chronic stress exposure may affect the brain's response to high calorie food cues and predispose to obesogenic eating habits. Physiol Behav 2013;120:233-242.

38. Bing X, Ming-Guo Q, et al. Alterations in the cortical thickness and the amplitude of low-frequency fluctuation in patients with post-traumatic stress disorder. Brain Res 2013; 1490:225-232.

39. Stefanovic B, Warnking JM, Pike GB. Hemodynamic and metabolic responses to neuronal inhibition. Neuroimage 2004; 22:771-778.

40. Yi Y, Xu FM, Xie HW. Correlation between the liver meridian and the frontal lobe in depression by needling at taichong (LV3): a resting-state fMRI study. Zhongguo Zhong Xi Yi Jie He Za Zhi 2011;31:1044-1050.
41. Yan CG, Zang YF. DPARSF: a MATLAB toolbox for "pipeline" data analysis of resting-state fMRI. Front Syst Neurosci 2011;4:13.

42. Frolich MA, Deshpande H, Ness T, et al. Quantitative changes in regional cerebral blood flow induced by cold, heat and ischemic pain: a continuous arterial spin labeling study. Anesthesiology 2012;117:857-867.

43. Celle S, Annweiler C, Pichot V, et al. Association between ambulatory 24-hour blood pressure levels and brain volume reduction: a cross-sectional elderly population-based study. Hypertension 2012;60:1324-1331.

44. Wang Y, Shen X, Ying J, et al. Pathologic analysis on hyperplasia of mammary gland with different syndromes based on infrared radiation temperature of acupoints. J Tradit Chin Med 2012;32:382-387.

45. Cho SY, Kim M, Sun JJ, et al. A comparison of brain activity between healthy subjects and stroke patients on fMRI by acupuncture stimulation. Chin J Integr Med 2013;19:269-276.

Address correspondence to: Yong Huang, PhD School of Traditional Chinese Medicine Southern Medical University 510515, Guangzhou Guangdong China

E-mail: nanfanglihuang@163.com 